for the manuscript

\title{
Electron Spin Relaxation Mechanisms of Atomic Hydrogen Trapped in Silsesquioxane Cages: the Role of Isotope Substitution
}

\author{
by \\ George Mitrikas ${ }^{* \dagger}$ and Raanan Carmieli ${ }^{\ddagger}$ \\ ${ }^{\dagger}$ Institute of Nanoscience and Nanotechnology, NCSR Demokritos, 15310 Athens, Greece \\ ${ }^{\ddagger}$ Department of Chemical Research Support, Weizmann Institute of Science, Rehovot 7610001, Israel \\ E-mail: g.mitrikas@inn.demokritos.gr
}

\section{Sample Preparation}

POSS synthesis: Octakis(trimethylsiloxy)silsesquioxane $\left(\mathrm{Si}_{8} \mathrm{O}_{12}\left(\mathrm{OSiMe}_{3}\right)_{8}\right.$ or $h_{72} \mathrm{Q}_{8} \mathrm{M}_{8}$, CAS 51777-38-9) and its deuterated analogue, $d_{72} \mathrm{Q}_{8} \mathrm{M}_{8}$, were prepared with a two-step route. In the first step, the octaanion $\left[\mathrm{Si}_{8} \mathrm{O}_{20}{ }^{8-}\right] \cdot 8\left[\mathrm{Me}_{4} \mathrm{~N}\right]^{+}$was synthesized according to [1]. $9 \mathrm{ml}(40 \mathrm{mmol})$ of TEOS (Fluka) were added dropwise in a $100 \mathrm{ml}$ round bottomed flask containing $14.3 \mathrm{ml}$ of $\mathrm{TMAOH} / \mathrm{H}_{2} \mathrm{O}(25 \%$ Merck $)$ and $24 \mathrm{ml} \mathrm{MeOH}$ and left for vigorous stirring for 3 days. After this reaction time, the mixture was concentrated by vacuum and cooled. Hydrated crystals of the octaanion were observed. These crystals were dehydrated after heating at $60{ }^{\circ} \mathrm{C}$ in high pressure vacuum. In the second step, $250 \mathrm{mg}$ of the dry octaanion powder were dissolved in $1 \mathrm{ml} \mathrm{MeOH}$ and the mixture was slowly added to a vigorously stirring mixture containing $0.5 \mathrm{ml}$ of trimethylchlorosilane (CAS 75-77-4, Aldrich) or trimethyl- $d_{9}$-chlorosilane (CAS 20395-57-7, Chiron) and $2.5 \mathrm{ml}$ of hexane pre-cooled with an ice bath at $0^{\circ} \mathrm{C}$. After 5 min stirring the ice bath was removed and the mixture was further stirred for $45 \mathrm{~min}$ at room temperature. The obtained clear solution was washed with water and hexane and the organic phase was collected. Organic solvents were removed using a rotary evaporator and the material was obtained as fine white powder. Both compounds were analyzed by FT-IR spectroscopy which identified characteristic resonances as shown in FIG.S1[2, 3].

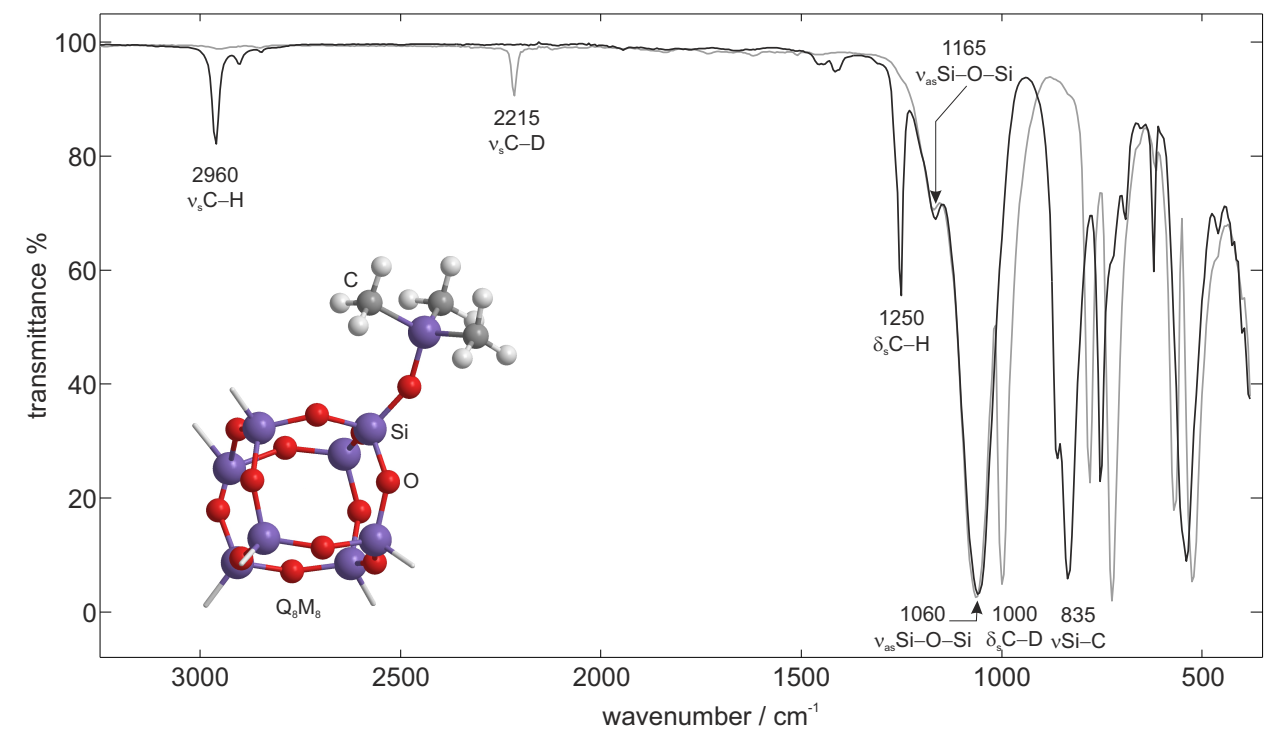

FIG. S1: FT-IR spectra of $h_{72} \mathrm{Q}_{8} \mathrm{M}_{8}$ (black trace) and $d_{72} \mathrm{Q}_{8} \mathrm{M}_{8}$ (gray trace). Inset: Molecular structure of $\mathrm{Si}_{8} \mathrm{O}_{12}\left(\mathrm{OSiMe}_{3}\right)_{8}\left(\mathrm{Q}_{8} \mathrm{M}_{8}\right)$, only one of the eight groups $\mathrm{R}$ is displayed; white balls denote $\mathrm{H}$ (for $h_{72} \mathrm{Q}_{8} \mathrm{M}_{8}$ ) or $\mathrm{D}$ (for $d_{72} \mathrm{Q}_{8} \mathrm{M}_{8}$ ) atoms.

Hydrogen encapsulation: Encapsulation of atomic hydrogen was performed with $\gamma$-irradiation in steps using a ${ }^{60} \mathrm{Co}$ source (dose rate of about $10 \mathrm{kGy} /$ day). The accumulated dose was measured using Red Perspex Dosimeters, Type 4034 AD. Typically $30 \mathrm{mg}$ of POSS powder were placed in a sealed vial with $\mathrm{O}_{2}$ 


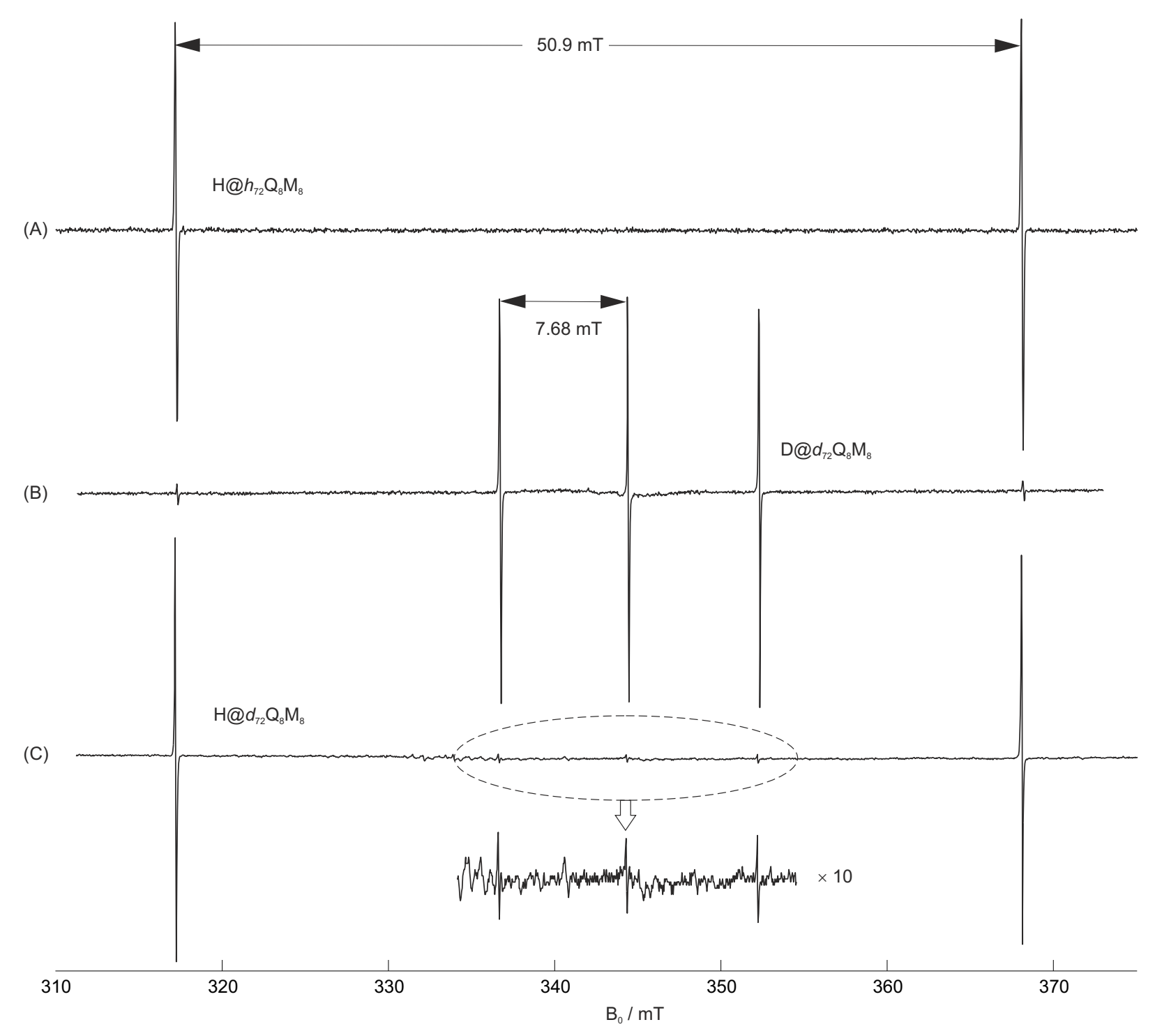

FIG. S2: X-band room-temperature EPR powder spectra of $\gamma$-irradiated samples with a dose of $200 \mathrm{kGy}$. Experimental conditions: microwave frequency, $9.615 \mathrm{GHz}$; microwave power attenuation, $40 \mathrm{~dB}$; modulation amplitude, $0.1 \mathrm{mT}$; modulation frequency, $100 \mathrm{kHz}$; number of accumulated spectra, 4

(acting as radical scavenger), and irradiated for 20 days resulting in a total dose of $200 \mathrm{kGy}$. Comparison of the $\mathrm{cw}$ EPR spectrum (in the presence of $\mathrm{O}_{2}$, unsaturated conditions) with a standard sample gave an estimate of 2.0-3.0 $\times 10^{16}$ spins per $\mathrm{cm}^{3}$ for the electron spin concentration. FIG.S2(A) and FIG.S2(B) illustrate the room-temperature EPR spectra of $\mathrm{H} @ h_{72} \mathrm{Q}_{8} \mathrm{M}_{8}$ and $\mathrm{D} @ d_{72} \mathrm{Q}_{8} \mathrm{M}_{8}$, respectively. A special $\gamma$ irradiation in solution was performed in order to inspect the encapsulation of hydrogen atoms originating from solvent molecules into the deuterated POSS cage. More specifically, $40 \mathrm{mg}$ of $d_{72} \mathrm{Q}_{8} \mathrm{M}_{8}$ were dissolved in $0.6 \mathrm{ml} \mathrm{C}_{6} \mathrm{H}_{14}$ and irradiated in a sealed vial under the same conditions as described above. After irradiation, the solvent was removed and the recovered powder was dried under vacuum $\left(10^{-1} \mathrm{mbar}\right)$ for 1 h. Interestingly, $\mathrm{H} @ d_{72} \mathrm{Q}_{8} \mathrm{M}_{8}$ turned to be the major occurring species (FIG.S2(C)), contrary to what was previously observed for $\gamma$-irradiation of $h_{72} \mathrm{Q}_{8} \mathrm{M}_{8}$ in $\mathrm{C}_{6} \mathrm{D}_{14}[4]$.

\section{2 cw-EPR results}

Fluid solution spectra: The ${ }^{29} \mathrm{Si}$ isotropic hyperfine couplings, $A_{29 \mathrm{Si}}$, were obtained from the analysis of the solution EPR spectra shown in FIG.S3. The spectra have narrow liewidths $\Delta B_{\mathrm{pp}}=12 \mu \mathrm{T}$ and exhibit satellite peaks separated by $64 \mu \mathrm{T}$ that are assigned to the hyperfine interaction between the unpaired electron and ${ }^{29} \mathrm{Si}$ atoms of the POSS cage. Assuming all eight positions are magnetically equivalent and taking into account the natural abundance $4.67 \%$ of ${ }^{29} \mathrm{Si}$, the abundance of POSS species with none (doublet, $\mathrm{s}_{1}$ ), one (quartet, $\mathrm{s}_{2}$ ), and two (sextet, $\mathrm{s}_{3}$ ) ${ }^{29} \mathrm{Si}$ atoms occurs from the binomial distribution as $68.15 \%$, 


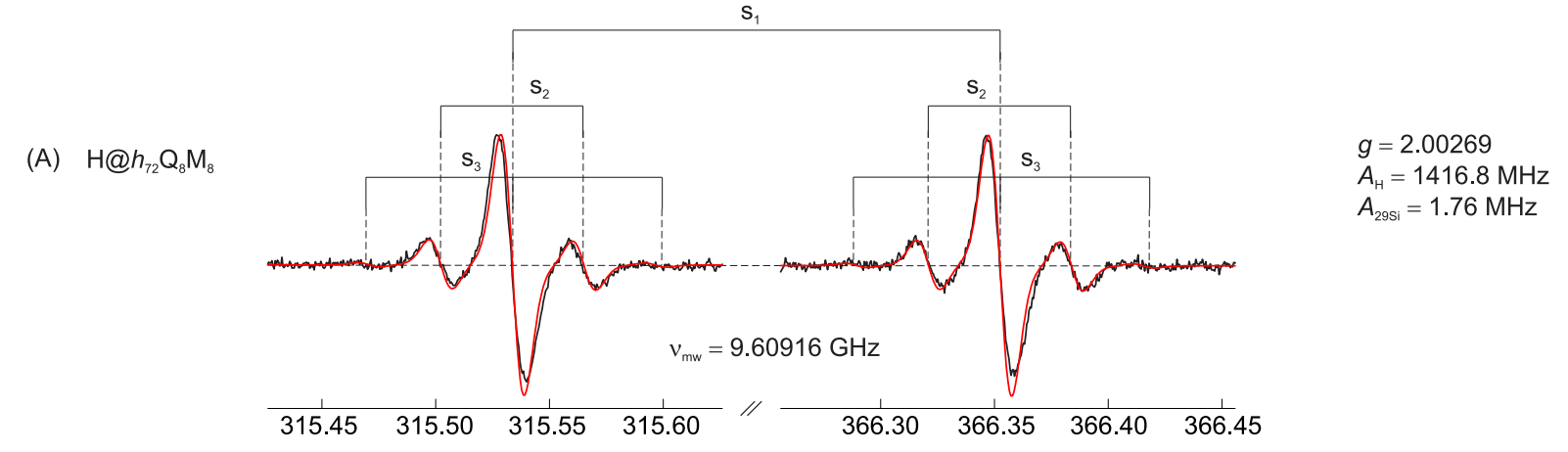

(B) $\mathrm{H} @ d_{72} \mathrm{Q}_{8} \mathrm{M}_{8}$

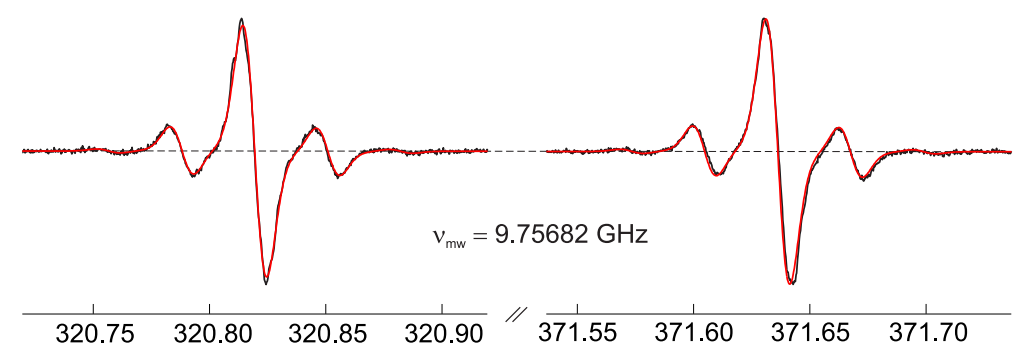

$g=2.00276$

$A_{\mathrm{H}}=1416.9 \mathrm{MHz}$

$A_{29 \mathrm{Si}}=1.76 \mathrm{MHz}$

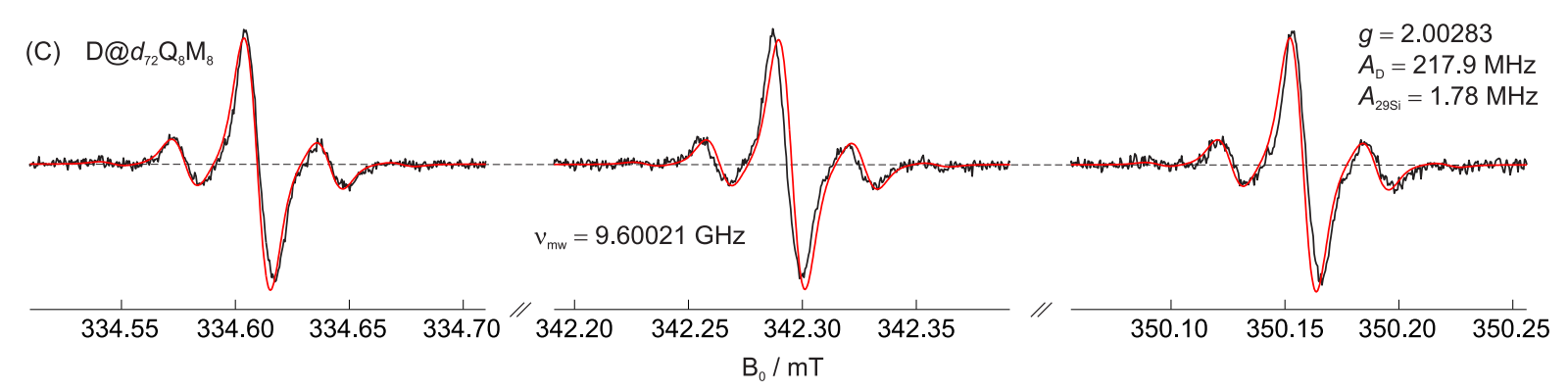

FIG. S3: Fluid solution spectra in degassed $n$-hexane (black traces) and the corresponding simulations (red traces) assuming isotropic H (or D) and ${ }^{29}$ Si hyperfine couplings and taking into account the natural abundance $4.67 \%$ of ${ }^{29} \mathrm{Si}$. Experimental conditions: mw attenuation, 40dB; modulation amplitude, $1 \mu \mathrm{T}$; modulation frequency, $100 \mathrm{kHz}$; number of accumulated spectra, 20

$26.79 \%$ and $4.58 \%$, respectively. These three species constitute more then $99.5 \%$ of the total paramagnetic centres and thus their properly weighted signals reproduce accurately the relative intensities of peaks in the experimental EPR spectrum as can be seen in FIG.S3. A nearly Gaussian lineshape with a FWHM of 11.5

(A) $\mathrm{H} @ d_{72} \mathrm{Q}_{8} \mathrm{M}_{8}$
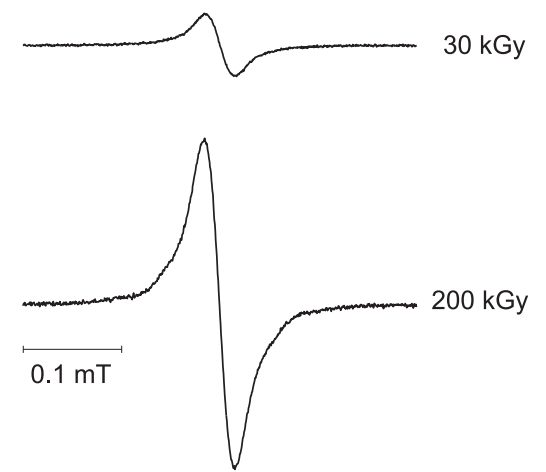

(B) $\mathrm{D} @ d_{72} \mathrm{Q}_{8} \mathrm{M}_{8}$
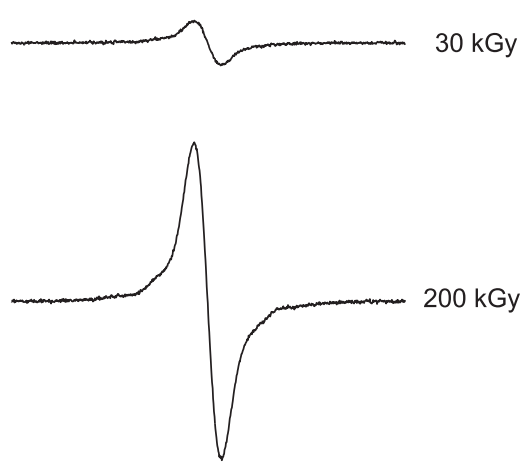

FIG. S4: X-band room-temperature EPR powder spectra of $\gamma$-irradiated $d_{72} \mathrm{Q}_{8} \mathrm{M}_{8}$ in solution (A) and in the solid state (B). Upper traces, $30 \mathrm{kGy}$; lower traces, $200 \mathrm{kGy}$. Spectra are scaled with the factor $m /(2 I+1)$, where $I$ is the nuclear spin number of the encapsulated atom. Experimental conditions: microwave power attenuation, $40 \mathrm{~dB}$; modulation amplitude, $5 \mu \mathrm{T}$; modulation frequency, $100 \mathrm{kHz}$; number of accumulated spectra, 20 
$\mu \mathrm{T}$ was used for the simulation which included convolution with a $4 \mu \mathrm{T}$ Lorentzian peak for better fit.

Low dose samples: In order to unravel the origin of the shorter $T_{\mathrm{M}}$ values observed for $\mathrm{H} @ d_{72} \mathrm{Q}_{8} \mathrm{M}_{8}$ at high temperatures (see Fig.5, main manuscript), we applied a lower $\gamma$-ray dose of $30 \mathrm{kGy}$ for both irradiations of $d_{72} \mathrm{Q}_{8} \mathrm{M}_{8}$ in the solid state and in solution. The mass $m$ of each sample was measured and the absolute number of contained spins was estimated by comparing the double integral of the EPR spectrum to that of a standard sample. The average spin concentration $C_{\mathrm{av}}$ for each sample was estimated assuming the density $d=1.16 \mathrm{~g} / \mathrm{cm}^{3}$. FIG.S4 illustrates the low-field EPR transitions of powder spectra for the four samples. To directly compare the different $C_{\mathrm{av}}$ values, the spectra intensities are scaled with the factor $m /(2 I+1)$.
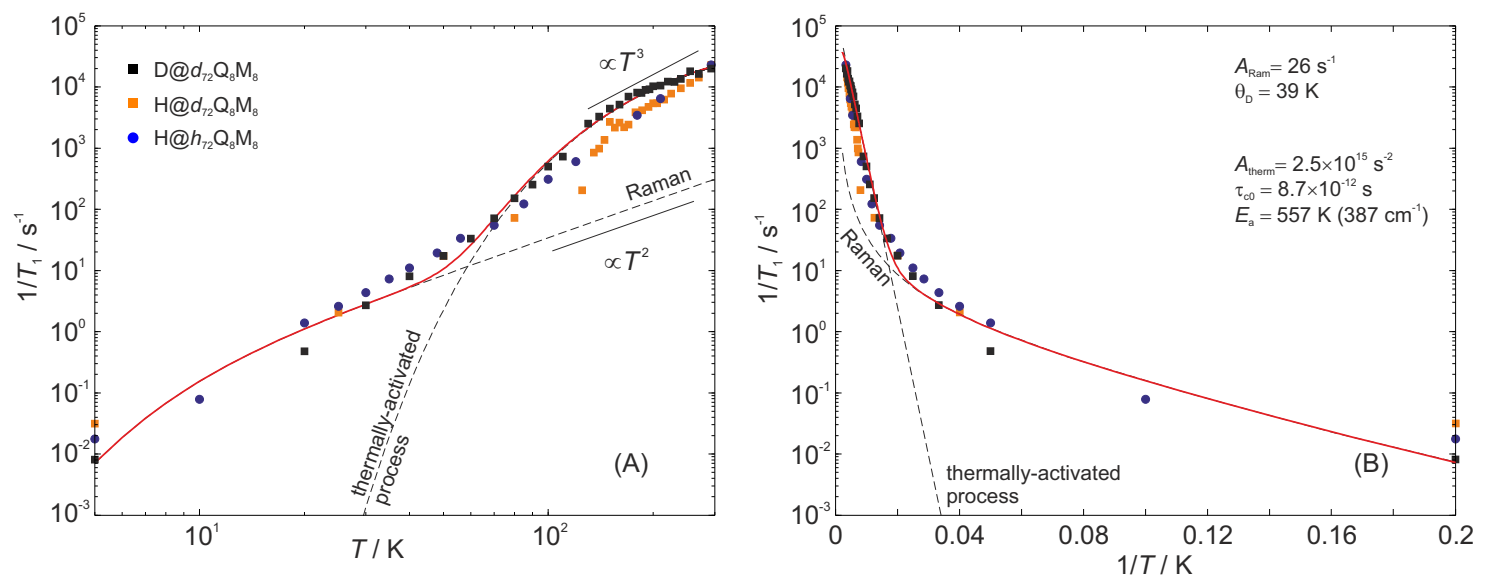

FIG. S5: Temperature dependence of the spin-lattice relaxation rate for the three samples in the "Raman" (A) and "Orbach" (B) representation. The red solid line represents the fitting curve to the data of $\mathrm{D} @ d_{72} \mathrm{Q}_{8} \mathrm{M}_{8}$ assuming contributions from Raman and thermally-activated processes (dashed lines) as described in [5].

\section{$3 \quad$ Pulse EPR results}

Spin-lattice relaxation: The temperature dependence of the spin-lattice relaxation rate $1 / T_{1}$ is displayed in FIG.S5. From the "Raman" $1 / T_{1}$ vs. $T \log -\log$ representation shown in FIG.S5(A) it is evident that more than one relaxation processes exist. At high temperatures the relaxation rate follows a $T^{3}$ dependence, stronger than the $T^{2}$ expected for a Raman process. This suggests that apart from the usual two-phonon there is at least one additional process that is characterized by a stronger than $T^{2}$ dependence at high temperatures, namely Orbach, local-mode, or thermally activated process as has been previously analyzed for similar species.[5, 6]

\section{Quantum rotational tunneling of methyl groups}

The rotation of methyl groups is usually modeled assuming a three-fold hindering potential of magnitude $V_{3}$ like the one shown in FIG.S6(A). The rotational Hamiltonian is described by

$$
\hat{\mathcal{H}}_{R}=-\frac{\hbar^{2}}{2 I} \frac{\partial^{2}}{\partial \theta^{2}}+V(\theta)
$$

where $I$ is the moment of inertia of $\mathrm{CH}_{3}$ and $V(\theta)=V_{3}(1-\cos (3 \theta+\pi)) / 2$. The librational levels can in first order be described by the the quantum harmonic oscillator, the exact solution is displayed in FIG.S6(A). Each one of them consists of a singlet (A) and a degenerate doublet (E) that are separated by the tunneling energy $h \nu_{t}$. The tunneling frequency $\nu_{t}$ depends approximately exponentially on the potential barrier $V_{3}$ and the rotor moment of inertia I. FIG.S6(B) displays the tunneling frequencies $\nu_{t}$ of the ground librational states for $\mathrm{CH}_{3}$ and $\mathrm{CD}_{3}$ calculated from the numerical solution of the Schrödinger equation (Eq.1). 


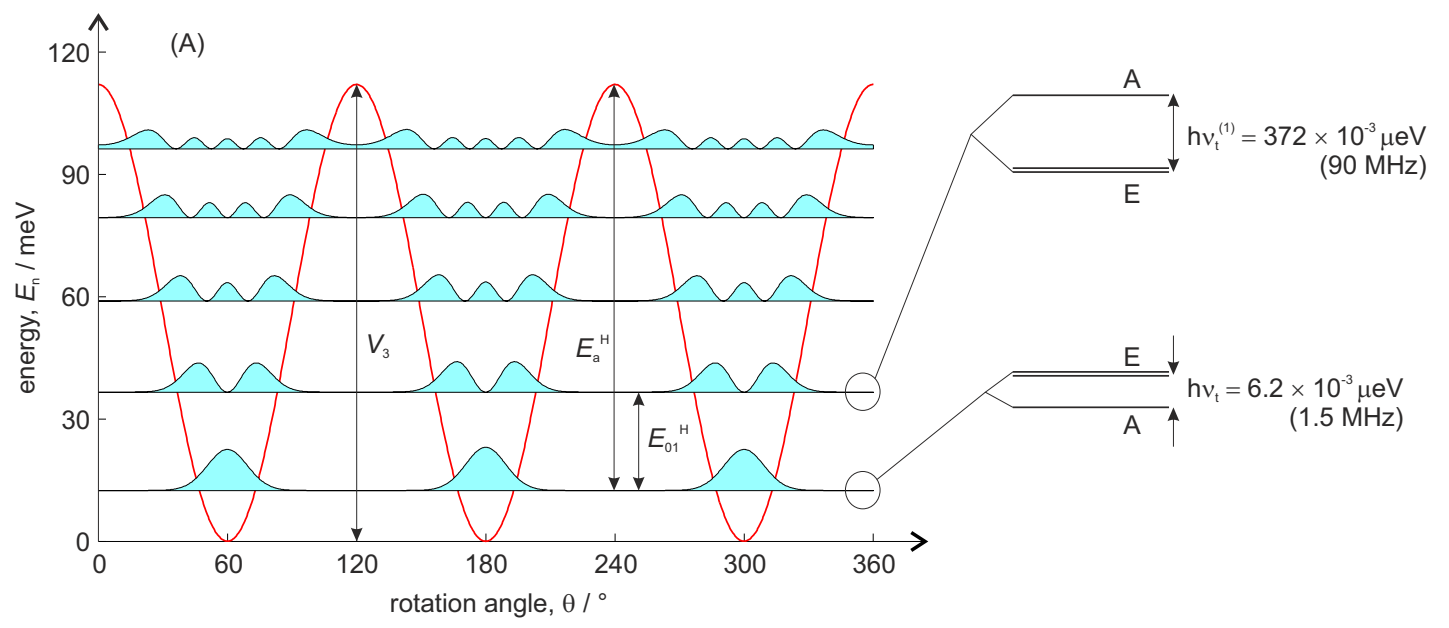

(B)

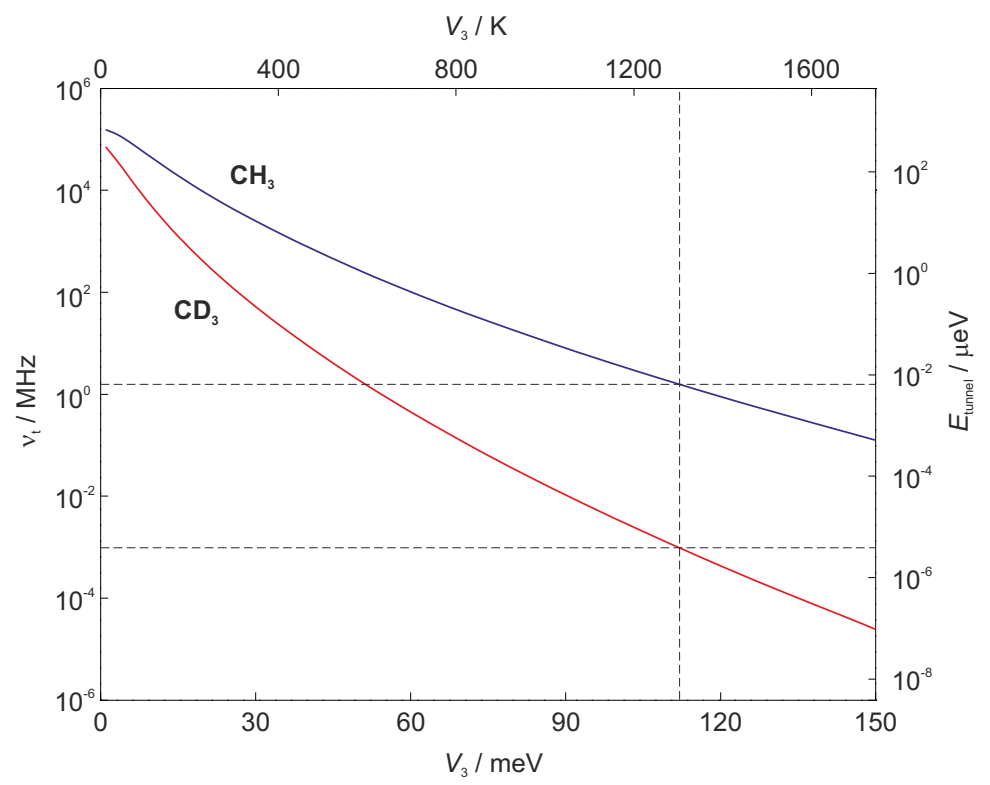

FIG. S6: (A) Schematic diagram of the $\mathrm{CH}_{3}$ librational energy levels of the three-fold potential with $V_{3}=112$ meV (1300 K). Shaded curves illustrate probability densities of the lowest five librational states. Right inset: enlargement showing the tunneling splitting of the lowest two states. (B) Tunneling splitting of the ground librational state as a function of $V_{3}$ for $\mathrm{CH}_{3}$ (blue trace) and $\mathrm{CD}_{3}$ (red trace).

\section{References}

[1] Y. Hagiwara, A. Shimojima, and K. Kuroda, Chem. Mater. 20, 1147 (2008).

[2] I. Hasegawa, W. Imamura, and T. Takayama, Inorg. Chem. Commun. 7, 513 (2004).

[3] R. Al-Oweini and H. El-Rassy, J. Mol. Struct. 919, 140 (2009).

[4] Y. Hayashino, T. Isobe, and Y. Matsuda, Inorg. Chem. 40, 2218 (2001).

[5] G. Mitrikas, Phys. Chem. Chem. Phys. 14, 3782 (2012).

[6] G. Mitrikas and S. Menenakou, Phys. Chem. Chem. Phys. 22, 15751 (2020). 